\title{
A HOFER-LIKE METRIC ON THE GROUP OF SYMPLECTIC DIFFEOMORPHISMS
}

\author{
Augustin BanYaga
}

\begin{abstract}
Using a "Hodge decomposition" of symplectic isotopies on a compact symplectic manifold $(M, \omega)$, we construct a norm on the identity component in the group of all symplectic diffeomorphisms of $(M, \omega)$ whose restriction to the group $\operatorname{Ham}(M, \omega)$ of hamiltonian diffeomorphisms is bounded from above by the Hofer norm. Moreover, $\operatorname{Ham}(M, \omega)$ is closed in $\operatorname{Symp}(M, \omega)$ equipped with the topology induced by the extended norm. We give an application to the $C^{0}$ symplectic topology. We also discuss extensions of Oh's spectral distance.
\end{abstract}

\section{Introduction and statement of the main results}

Let $\operatorname{Symp}(M, \omega)$ denote the group of all symplectic diffeomorphisms of a compact symplectic manifold $(M, \omega)$, endowed with the $C^{\infty}$ compact-open topology, and $\operatorname{Symp}(M, \omega)_{0}=G_{\omega}(M)$ the identity component in $\operatorname{Symp}(M, \omega) . \operatorname{Symp}(M, \omega)_{0}$ consists of symplectic diffeomorphisms $h$ such that there is a symplectic isotopy $h_{t}$ from the identity to $h$. By definition $h_{t}$ is a symplectic isotopy if the map $(x, t) \mapsto h_{t}(x)$ is smooth and for all t , $h_{t}^{*} \omega=\omega$. We denote by $I s o(M)$ the set of all symplectic isotopies, and by $I s o(\phi)$ the set of all symplectic isotopies from the identity to $\phi \in \operatorname{Symp}(M, \omega)_{0}$.

Let $\operatorname{Ham}(M, \omega) \subset \operatorname{Symp}(M, \omega)_{0}$ be the subgroup of Hamiltonian diffeomorphisms. A diffeomorphism $\psi$ is Hamiltonian iff it is the time 1 map of a smooth family of diffeomorphisms $\psi_{t}$ such that if

$$
\dot{\psi}_{t}(x)=\frac{d \psi_{t}}{d t}\left(\psi_{t}^{-1}(x)\right), \psi_{0}(x)=x
$$

1991 Mathematics Subject Classification. MSC2000:53D05; 53D35.

Key words and phrases. Harmonic diffeomorphism, harmonic vector field, Hofer metric, spectral norm, the flux conjecture, $C^{0}$-symplectic topology. 
there exists a smooth family of functions $u_{t}$ such that

$$
i_{\left(\dot{\psi}_{t}\right)} \omega=d u_{t}
$$

The family of diffeomorphisms $\psi_{t}$ above is called a hamiltonian isotopy.

We denote by $\operatorname{HIso}(\phi)$ the set of all hamiltonian isotopies from $\phi \in \operatorname{Ham}(M, \omega)$ to the identity, and by $H I s o(M)$ the set of all hamiltonian isotopies.

In equation $(2), i_{(.)}$denotes the interior product: $i_{X} \omega$ is the 1 -form such that $i_{X} \omega(Y)=\omega(X, Y)$. Recall that a symplectic form is a closed 2-form $\omega$ such that the map assigning to a vector field $X$ the 1 -form $i_{X} \omega$ is an isomorphism $\tilde{\omega}$. For any 1-form $\alpha$, we denote by $\alpha^{\#}$ the vector field $\tilde{\omega}^{-1}(\alpha)$.

The Hofer length of a hamiltonian isotopy $\psi_{t}$ is defined as:

$$
l_{H}\left(\psi_{t}\right)=\int_{0}^{1}\left(\max _{x} u_{t}(x)-\min _{x} u_{t}(x)\right) d t
$$

One also denotes

$$
\left.\max _{x} u_{t}(x)-\min _{x} u_{t}(x)\right)=\operatorname{osc}\left(u_{t}(x)\right)
$$

and call it the oscillation of $u_{t}$.

Hence the Hofer length is the mean oscillation of the hamiltonian $u_{t}$ of the hamiltonian isotopy $\Phi=\left(\phi_{t}\right)$.

For $\psi \in \operatorname{Ham}(M, \omega)$, the Hofer norm is defined as:

$$
\|\psi\|_{H}=\inf \left(l_{H}\left(\psi_{t}\right)\right)
$$

where the infimum is taken over all hamiltonian isotopies $\psi_{t} \in H I s o(\psi)$ and $u_{t}$ is the function in equation (2).

The Hofer distance between two hamiltonian diffeomorphisms $\phi$ and $\psi$ is:

$$
d_{H}(\phi, \psi)=\left\|\phi \psi^{-1}\right\|_{H}
$$


It is easy to see that the formula above defines a bi-invariant pseudo-metric but it is very challenging to show that it is not degenerate and hence it is a genuine distance [5],[7],[12], [13].

In this paper we propose a formula for the length of a symplectic isotopy $\Phi=\left(\phi_{t}\right)$ (5), which generalizes the length of a hamiltonian isotopy (3).

Fix a riemannian metric on $M$ and consider the Hodge decomposition of $i_{\left(\dot{\phi}_{t}\right)} \omega$

$$
i_{\left(\dot{\phi}_{t}\right)} \omega=\mathcal{H}_{t}^{\Phi}+d u_{t}^{\Phi}
$$

where $\mathcal{H}_{t}^{\Phi}$ and $u_{t}^{\Phi}$ are smooth family of harmonic 1-forms and functions respectively.

We define the length $l(\Phi)$ of the isotopy $\Phi$ by:

$$
l(\Phi)=\int_{0}^{1}\left(\left|\mathcal{H}_{t}^{\Phi}\right|+\left(\max _{x}\left(u_{t}^{\Phi}\right)-\min _{x}\left(u_{t}^{\Phi}\right)\right) d t\right.
$$

Here $\left|\mathcal{H}_{t}^{\Phi}\right|$ is the "Euclidean" norm of the harmonic 1-form $\mathcal{H}_{t}^{\Phi}$ ( see (13), (14)).

This formula reduces to (3) for hamiltonian isotopies. Unfortunately, unlike (3), we do not have

$$
l(\Phi)=l\left(\Phi^{-1}\right)
$$

where $\Phi^{-1}=\left(\phi_{t}^{-1}\right)$. We will also write:

$$
\left.l(\Phi)=\| \dot{\phi}_{t}\right) \|
$$

For any $\phi \in \operatorname{Symp}(M, \omega)_{0}$, we define the energy $e_{0}(\phi)$ of $\phi$ as:

$$
e_{0}(\phi)=i n f_{\Phi \in I s o(\phi)}(l(\Phi))
$$

Our main result is the following 


\section{Theorem 1.}

Let $(M, \omega)$ be a closed symplectic manifold. Consider the map e $: \operatorname{Symp}(M, \omega)_{0} \rightarrow$ $\mathbb{R} \cup\{\infty\}$

$$
e(\phi)=1 / 2\left(e_{0}(\phi)+e_{0}\left(\phi^{-1}\right)\right) .
$$

Then e is a norm on $\operatorname{Symp}(M, \omega)_{0}$ whose restriction to $\operatorname{Ham}(M, \omega)$ is bounded from above by the Hofer metric.

Moreover the subgroup $\operatorname{Ham}(M, \omega)$ is closed in $\operatorname{Symp}(M, \omega)$ endowed with the metric topology defined by e.

We define a distance on $\operatorname{Symp}(M, \omega)$ by:

$$
d(\phi, \psi)=e\left(\phi \psi^{-1}\right)
$$

This distance is obviously right invariant, but not left invariant.

\section{Remark}

The fact that (5) reduces to (3) when $\Phi$ is a hamiltonian isotopy implies that

$$
e(\phi) \leq\|\phi\|_{H}
$$

for all $\phi \in \operatorname{Ham}(M, \omega)$.

Conjecture The restriction of the norm $e$ to $\operatorname{Ham}(M, \omega)$ is equivalent to the Hofer norm.

What is the interest of our construction? From the Hofer norm, there are easy ways of constructing bi-invariant norms on $\operatorname{Symp}(M, \omega)$. One is given by Han [4]:

fix a positive number $K$ and define

$$
\|\phi\|_{K}= \begin{cases}\min \left(\|\phi\|_{H}, K\right), & \text { if } \phi \in \operatorname{Ham}(M, \omega) \\ K & \text { otherwise }\end{cases}
$$

Another is given by Lalonde-Polterovich [8]:

fix a real number $\alpha$ and define

$$
\|\phi\|_{\alpha}=\sup \left\{\left\|\phi f \phi^{-1} f^{-1}\right\|_{H} \mid f \in \operatorname{Ham}(M, \omega),\|f\|_{H} \leq \alpha\right\} .
$$


In both cases the restriction of these metrics back to $\operatorname{Ham}(M, \omega)$ gives different topologies on $\operatorname{Ham}(M, \omega)$. In particular $\operatorname{Ham}(M, \omega)$ in these topology has always a finite diameter which is known to be untrue for the Hofer norm in several cases.

Hence the advantage of our construction is that its restriction to $\operatorname{Ham}(M, \omega)$ gives a "better" topology, which may be the same if the conjecture is true.

Moreover the "Hofer-like" formula (5) allows to define a distance $D$ on the space Iso $(M)$ of symplectic isotopies of $(M, \omega)$. If $\Phi=\left(\phi_{t}\right)$ and $\Psi=\left(\psi_{t}\right)$ are symplectic isotopies define:

$$
D(\Phi, \Psi)=\left\|\dot{\phi}_{t}-\dot{\psi}_{t}\right\|=: \int_{0}^{1}\left(\left|\mathcal{H}^{\Phi_{t}}-\mathcal{H}^{\Psi_{t}}\right|+o s c\left(u^{\Phi_{t}}-u^{\Psi_{t}}\right)\right) d t
$$

It is clear that $D$ is a distance. Moreover if $\Phi, \Psi$ are hamiltonian isotopies, then

$$
D(\Phi, \Psi)=l_{H}\left(\Phi \Psi^{-1}\right)
$$

In general the formula above is not true.

The distance formula will be used to the define the symplectic topology on $I s o(M)$.

\section{Hamiltonian and harmonic diffeomorphisms}

For each symplectic isotopy $\Phi=\left(\phi_{t}\right)$, consider the following 1-form:

$$
\Sigma(\Phi)=\int_{0}^{1}\left(i_{\dot{\phi}_{t}} \omega\right) d t
$$

It is shown in [1], ( see also [2]) that the cohomology class $\left[\Sigma(\Phi] \in H^{1}(M, \mathbb{R})\right.$ of the form $\Sigma(\Phi)$ depends only on the class $[\Phi]$ of $\Phi$ in the universal covering $\tilde{G}(M, \omega)$ of $\operatorname{Symp}(M, \omega)_{0}=G(M, \omega)$ and that the map $[\Phi] \mapsto[\Sigma(\Phi)]$ is a surjective homomorphism

$$
\tilde{S}: \tilde{G}(M, \omega) \rightarrow H^{1}(M, \mathbb{R})
$$

The group

$$
\Gamma=\tilde{S}\left(\pi_{1}(G(M, \omega))\right) \subset H^{1}(M, \mathbb{R})
$$

is called the flux group. 
In [1], it was observed that $\Gamma$ was discrete in several examples and the author wrote "I do not know any flux group which is not discrete". The statement that " $\Gamma$ is discrete" became known as the "Flux conjecture". This conjecture has been recently proved by Ono [11] using Floer-Novikov homology.

\section{Theorem (Ono).}

Let $(M, \omega)$ be a compact symplectic manifold, then the flux group is discrete.

Consider the induced homomorphism :

$$
S: G(M, \omega) \rightarrow H^{1}(M, \mathbb{R}) / \Gamma
$$

In [1], [2], it is shown that the Kernel of S coincides with the group $\operatorname{Ham}(M, \omega)$ of Hamiltonian diffeomorphisms, and it is a simple group, which coincides with the commutator subgroup $[G(M, \omega), G(M, \omega)]$ of $G(M, \omega)$. We summarize:

$$
\operatorname{Ham}(M, \omega)=\operatorname{Ker} S=[G(M, \omega), G(M, \omega)]
$$

for all closed symplectic manifolds $(M, \omega)$.

We will need to represent in a unique way cohomology classes ; this is achieved by Hodge theory on compact riemannian manifolds.

The Hodge decomposition theorem ( see for instance [14]) asserts that any smooth family of p-forms $\theta_{t}$ on a compact oriented riemannian manifold $(M, g)$ can be decomposed in a unique way as

$$
\theta_{t}=\mathcal{H}_{t}+d \alpha_{t}+\delta \beta_{t}
$$

where $\mathcal{H}_{t}$ is harmonic, i.e $d \mathcal{H}_{t}=\delta \mathcal{H}_{t}=0$. Here $\delta$ denotes the codifferential. If $d \theta_{t}=0$, then $\delta \beta_{t}=0$. The forms $\mathcal{H}_{t}, \alpha_{t}$ and $\beta_{t}$ depend smoothly on $t$.

The harmonic form $\mathcal{H}_{t}$ is a unique representative of the cohomology class $\left[\theta_{t}\right] \in$ $H^{1}(M, \mathbb{R})$ of $\theta_{t}$. 


\section{Definition 1.}

Let $(M, \omega)$ be a compact symplectic manifold, equipped with some riemannian metric. A vector field $X$ on $M$ is said to be a harmonic vector field if $i_{X} \omega$ is a harmonic form.

A diffeomorphism $\phi$ of $M$ is said to be a harmonic diffeomorphism if if there exists a smooth family $\mathcal{H}_{t}$ of harmonic 1-forms such that $\phi$ is the time 1 map of the symplectic isotopy $\phi_{t}$ such that

$$
\dot{\phi}_{t}=\left(\mathcal{H}_{t}\right)^{\#} \text {. }
$$

We say that $\phi_{t}$ is a harmonic isotopy.

Let $\operatorname{symp}(M, \omega)$ be the set of symplectic vector fields, $\operatorname{harm}(M)$ the set of harmonic vector fields and $\operatorname{ham}(M, \omega)$ the space of hamiltonian vector fields. If $X \in \operatorname{symp}(M, \omega)$ then $i_{X} \omega$ is closed. The decomposition $i_{X} \omega=\mathcal{H}+d u$ expresses $X$ as

$$
X=H+X_{u}
$$

where $H=(\mathcal{H})^{\#}$ is harmonic and $X_{u}$ is the hamiltonian vector field with hamiltonian $u$.

Hence $\operatorname{symp}(M, \omega)$ is the Cartesian product of $\operatorname{harm}(M)$ and $\operatorname{ham}(M, \omega)$. We give $\operatorname{symp}(M, \omega)$ the product metric :

$$
|X|=|H|+\max _{x} u(x)-\min _{x} u(x)
$$

where $|H|$ is the norm on $\operatorname{harm}(M)$ given below:

the space $\operatorname{harm}(M)$, which is isomorphic to the space of harmonic 1-forms is a finite dimensional vector space whose dimension is the first Betti number of $M$.

In this paper, we fix a basis $h_{1}, \ldots, h_{r}$ of harmonic 1 -forms and consider $\left(H_{i}\right)=$

$\left(h_{i}^{\#}\right)$ the corresponding basis of $\operatorname{harm}(M)$. We give these 2 vector spaces the following Euclidean metric : if $h=\sum_{i} \lambda_{i} h_{i}, H=\sum_{i} \lambda_{i} H_{i}$, then

$$
|h|=|H|=\sum_{i}\left|\lambda_{i}\right|
$$


In view of (13), the length formula (5) gives a Finsler metric on $\operatorname{Symp}(M, \omega)$.

\section{Remark}

The function $u$ in the Hodge decomposition $i_{X} \omega=\mathcal{H}+d u$ is not necessarly normalized. However if in (13) $|X|=0$, then $|H|=0$, i.e $i_{X} \omega=d u$ and $\operatorname{osc}(u)=0$ implies that $u$ is constant, and hence $d u=0$. Therefore $X=0$.

\section{Lemma 1.}

Any symplectic isotopy $\Phi=\left(\phi_{t}\right)$ on a compact symplectic manifold $(M, \omega)$ can be decomposed in a unique way as

$$
\phi_{t}=\rho_{t} \cdot \psi_{t}
$$

where $\rho_{t}$ is a harmonic isotopy and $\psi_{t}$ is a hamiltonian isotopy. In particular, if $\phi_{t}$ is a hamiltonian isotopy, then $\phi_{t}=\psi_{t}$ and $\rho_{t}=i d_{M}$.

Proof.

By Hodge decomposition theorem $i_{\left(\dot{\phi}_{t}\right)} \omega$ can be decomposed in a unique way as

$$
i_{\left(\dot{\phi}_{t}\right)} \omega=\mathcal{H}_{t}^{\Phi}+d u_{t}^{\Phi}
$$

where $\mathcal{H}_{t}^{\Phi}$ and $u_{t}^{\Phi}$ are smooth family of harmonic 1-forms and functions respectively. Let $\rho_{t}$ be the harmonic isotopy such that $\dot{\rho}_{t}=\left(\mathcal{H}_{t}\right)^{\#}$. Set now $\psi_{t}=\left(\rho_{t}\right)^{-1} \cdot \phi_{t}$. From $\phi_{t}=\rho_{t} \cdot \psi_{t}$, we get:

$$
\dot{\phi}_{t}=\dot{\rho}_{t}+\left(\rho_{t}\right)_{*} \dot{\psi}_{t}
$$

Since $i_{\left(\dot{\phi}_{t}-\dot{\rho}_{t}\right)} \omega=d u_{t}=i_{\left(X_{\left(u_{t}\right)}\right)} \omega$ where $X_{u_{t}}$ is the hamiltonnian vector field of $u_{t}$, we see that

$$
\dot{\phi}_{t}=\dot{\rho}_{t}+X_{u_{t}}=\dot{\rho}_{t}+\left(\rho_{t}\right)_{*}\left(\left(\rho_{t}\right)^{-1}\right)_{*}\left(X_{u_{t}}\right)
$$

Hence $\left.\dot{\psi}_{t}=\left(\rho_{t}\right)^{-1}\right)_{*}\left(X_{u_{t}}\right)=X_{\left(u_{t} \circ \rho_{t}\right)}$. This shows that $\psi_{t}$ is a hamiltonnian isotopy. 
In formula (5), $\int_{0}^{1} \operatorname{osc}\left(u_{t}^{\Phi}\right) d t$ is nothing else than $l_{H}\left(\psi_{t}\right)$ and formula (5) can be written

$$
\left.l(\Phi)=\int_{0}^{1}\left|i\left(\dot{\rho}_{t}\right) \omega\right| d t+l_{H}\left(\psi_{t}\right)\right) d t
$$

\section{Prooof of theorem 1}

Clearly, $e(\phi) \geq 0$ for all $\phi$ and by definition $e(\phi)=e\left(\phi^{-1}\right)$.

To see that the triangular inequality holds, fix a small positive number $\epsilon \leq 1 / 8$ and a smooth increasing function $a:[0,1] \rightarrow[0,1]$ such that $a_{\mid[0, \epsilon)}=0$ and a $a_{\mid(1-\epsilon), 1]}=1$ and let $\lambda(t)=a(2 t)$ for $0 \leq t \leq 1 / 2$ and $\mu(t)=a(2 t-1)$ for $1 / 2 \leq t \leq 1$.

If $\Phi \in I \operatorname{so}(\phi)$ and $\Psi \in I s o(\psi)$, we get an isotopy $\Phi * \Psi=\left(\sigma_{t}\right) \in I s o(\phi \psi)$ defined as:

$$
\sigma_{t}= \begin{cases}\phi_{\lambda(t)}, & \text { for } 0 \leq t \leq 1 / 2 \\ \phi_{1} \psi_{\mu(t)}, & \text { for } 1 / 2 \leq t \leq 1\end{cases}
$$

Let $c(\Phi, \Psi)$ be the set of all isotopies from $\phi \psi$ to the identity obtained as above.

Clearly :

$$
e_{0}(\phi \psi) \leq i n f_{\mathcal{R}}(l(\mathcal{R}))
$$

where $\mathcal{R} \in c(\Phi, \Psi)$.

Since

$$
\dot{\sigma}_{t}= \begin{cases}\lambda^{\prime} \dot{\phi}_{\lambda(t)}, & \text { for } 0 \leq t \leq 1 / 2 \\ \mu^{\prime} \dot{\psi}_{\mu(t)}, & \text { for } 1 / 2 \leq y \leq 1\end{cases}
$$

we have:

$$
i\left(\dot{\sigma}_{t}\right) \omega= \begin{cases}\lambda^{\prime} \mathcal{H}_{\lambda(t)}^{\Phi}+d\left(\lambda^{\prime} u_{\lambda(t)}^{\Phi}\right) & \text { for } 0 \leq t \leq 1 / 2 \\ \mu^{\prime} \mathcal{H}_{\mu(t)}^{\Phi^{\prime}}+d\left(\mu^{\prime} u_{\mu(t)}^{\Phi^{\prime}}\right), & \text { for } 1 / 2 \leq t \leq 1\end{cases}
$$

Therefore

$l(\Phi * \Psi)=\int_{0}^{1 / 2}\left(\left|\lambda^{\prime} \mathcal{H}_{\lambda(t)}^{\Phi}\right|+\operatorname{osc}\left(\lambda^{\prime} u_{\lambda(t)}^{\Phi}\right) \mid\right) d t+\int_{1 / 2}^{1}\left(\left|\mu^{\prime} \mathcal{H}_{\mu(t)}^{\Phi^{\prime}}\right|+o s c\left(\mu^{\prime} u_{\mu(t)}^{\Phi^{\prime}}\right) d t\right.$ 
By the change of variable formula, we get:

$$
l(\Phi * \Psi)=l(\Phi)+l(\Psi)
$$

Finally,

$$
e_{0}(\phi \psi) \leq i n f_{\mathcal{R}}(l(\mathcal{R})) \leq i n f_{\Phi} l(\Phi)+i n f_{\Psi} l(\Psi)=e_{0}(\phi)+e_{0}(\psi)
$$

Therefore the triangular inequality holds true for $e_{0}$, and hence for $e$ as well.

Showing that $e$ is non-degenerate is more delicate. Suppose that $e_{0}(\phi)=0$.

\section{Step 1}

The statement $e_{0}(\phi)=\inf (l(\Phi))=0$ means that for every $N$, there exists an isotopy $\Phi^{N}$ from $\phi$ to the identity such that $l\left(\Phi^{N}\right) \leq 1 / N$.

Thus:

$$
\int_{0}^{1}\left|\mathcal{H}_{t}^{\Phi^{N}}\right| d t \leq 1 / N
$$

and

$$
\int_{0}^{1} \operatorname{osc}\left(u^{\Phi^{N}}\right) d t \leq 1 / N
$$

Hence

$$
\left|\mathcal{H}\left(\Phi^{N}\right)\right|=\left|\int_{0}^{1} \mathcal{H}_{t}^{\Phi^{N}} d t\right| \leq \int_{0}^{1}\left|\mathcal{H}_{t}^{\Phi^{N}}\right| d t \leq 1 / N
$$

For any symplectic isotopy from $\phi$ to the identity $\Phi=\left(\phi_{t}\right)$, the 1 -form

$$
\mathcal{H}(\Phi)=\int_{0}^{1} \mathcal{H}_{t}^{\Phi} d t
$$

is the harmonic representative of the cohomology class $\tilde{S}\left(\left[\phi_{t}\right]\right)$.

For any symplectic isotopy $\Phi=\left(\phi_{t}\right)$ from $\phi$ to the identity

$$
\mathcal{H}\left(\Phi^{N}\right)-\mathcal{H}(\Phi)=\gamma(\Phi) \in \Gamma
$$

since $\mathcal{H}\left(\Phi^{N}\right)-\mathcal{H}(\Phi)$ is the harmonic representative of the image by $\tilde{S}$ of the class $\left[\phi_{t}^{N} * \phi_{(1-t)}\right]$ of the loop $\phi_{t}^{N} * \phi_{(1-t)}$. 
By (16) and (17), the distance $d(\mathcal{H}(\Phi), \Gamma)$ from $\mathcal{H}(\Phi)$ to $\Gamma$ satisfies:

$$
d(\mathcal{H}(\Phi), \Gamma) \leq \mid \mathcal{H}(\Phi)-\left(-\gamma\left(\Phi^{N}\right)|=| \mathcal{H}\left(\Phi^{N}\right) \mid \leq 1 / N\right.
$$

This says that $(\mathcal{H}(\Phi))$ is arbitrarly close to $\Gamma$. Hence $(\mathcal{H}(\Phi)) \in \Gamma$. This means that $\phi \in \operatorname{Ker} S=\operatorname{Ham}(M, \omega)$.

The facts that $\mathcal{H}\left(\Phi^{N}\right) \in \Gamma$ and $\left|\mathcal{H}\left(\Phi^{N}\right)\right| \leq 1 / N$ imply that $\mathcal{H}\left(\Phi^{N}\right)=0$ for $N$ large enough since $\Gamma$ is discrete (Ono's theorem).

Fix now an isotopy $\Phi^{N}$ such that $\mathcal{H}\left(\Phi^{N}\right)=0$. To simplify the notations, we denote by $\Phi=\left(\phi_{t}\right)$ the isotopy $\Phi^{N}=\left(\phi_{t}^{N}\right)$.

The Hodge decomposition of the isotopy $\phi_{t}$ gives:

$$
\phi_{t}=\rho_{t} \mu_{t}
$$

where $\rho_{t}$ is harmonic and $\mu_{t}$ is hamiltonian. We have:

$$
\begin{gathered}
i\left(\dot{\phi}_{t}\right) \omega=\mathcal{H}_{t}+d u_{t} \\
\dot{\rho}_{t}=\left(\mathcal{H}_{t}\right)^{\#}=H_{t}, \\
\int_{0}^{1} \mathcal{H}_{t} d t=0
\end{gathered}
$$

and

$$
\int_{0}^{1}\left(\left|\mathcal{H}_{t}\right|+\operatorname{osc}\left(u_{t}\right)\right) d t \leq 1 / N
$$

Hence

$$
\int_{0}^{1}\left|\mathcal{H}_{t}\right| d t \leq 1 / N ; \int_{0}^{1} \operatorname{osc}\left(u_{t}\right) d t \leq 1 / N
$$

\section{Step 2}

We are now going to deform the isotopy $\rho_{t}$ fixing the extremities to a hamiltonian isotopy following [1], proposition II.3.1.

Let $Z_{(s, t)}$ be the family of symplectic vector fields:

$$
\left.Z_{(s, t)}=t \dot{\rho}_{(s . t)}-2 s\left(\int_{0}^{t}\left(i\left(\dot{\rho}_{u}\right) \omega\right) d u\right)\right) \# .
$$


Clearly, $Z_{(0, t)}=0$ and we have:

$$
\int_{0}^{1} i\left(Z_{(s, t)}\right) \omega d s=0
$$

Let $G_{(s, t)}$ be the 2-parameter family of diffeomorphisms obtained by integrating $Z_{(s, t)}$ with $t$ fixed, i.e. $G_{(s, t)}$ is defined by the following equations:

$$
\frac{d}{d s} G_{(s, t)}(x)=Z_{(s, t)}\left(G_{(s, t)}^{-1}(x)\right), G_{(0, t)}(x)=x .
$$

By $(20), G_{(1, t)}$ is a hamiltonnian diffeomorphism for all $t$. Since $Z_{(s, 1)}=\dot{\rho}_{s}-$ $2 s\left(\left(\int_{0}^{1}\left(i\left(\dot{\rho}_{u}\right) \omega\right) d u\right)=\dot{\rho}_{s}, s \mapsto G_{(s, 1)}\right.$ is an isotopy from the identity to $G(1,1)=\rho_{1}$. Hence the $g_{t}=G_{(1, t)}$ is an isotopy in $\operatorname{Ham}(M, \omega)$ from $\rho_{1}$ to the identity.

Consider the 2-parameter family of vector fields $V_{(s, t)}$ defined by:

$$
V_{(s, t)}(x)=\frac{d}{d t} G_{(s, t)}\left(\left(G_{(s, t)}^{-1}(x)\right)\right.
$$

Clearly $\dot{g}_{t}=V_{(1, t)}$.

We have ( see [1], proposition I.1.1):

$$
\frac{\partial}{\partial s} V_{(s, t)}=\frac{\partial}{\partial t} Z_{(s, t)}+\left[V_{(s, t)}, Z_{(s, t)}\right]
$$

We will need the following

\section{Proposition.}

$$
i\left(V_{(1, t)}\right) \omega=d u_{t}
$$

where $u_{t}=\int_{0}^{1} \omega\left(Z_{(s, t)}, V_{(s, t)}\right) d s$.

Proof.

¿From equation 22

$$
0=\frac{\partial}{\partial t}\left[\int_{0}^{1} i\left(Z_{(s, t)}\right) \omega d s\right]=\int_{0}^{1} i\left(\frac{\partial}{\partial t}\left(Z_{(s, t)}\right)\right) \omega d s
$$


A HOFER-LIKE METRIC ON THE GROUP OF SYMPLECTIC DIFFEOMORPHISMS 13

$$
\begin{aligned}
& =\int_{0}^{1} i\left(\frac{\partial}{\partial s}\left(V_{(s, t)}\right)\right) \omega d s-\int_{0}^{1} i\left(\left[Z_{(s, t)}, V_{(s, t)}\right]\right) \omega d s=\int_{0}^{1}\left(\frac{\partial}{\partial s} i\left(V_{(s, t)}\right) \omega\right) d s-\int_{0}^{1} i\left(\left[Z_{(s, t)}, V_{(s, t)}\right]\right) \omega d s \\
& =i\left(V_{(1, t)}\right) \omega-i\left(V_{(0, t)}\right) \omega-\int_{0}^{1} i\left(\left[Z_{(s, t)}, V_{(s, t)}\right]\right) \omega d s=i\left(V_{(1, t)}\right) \omega-d\left(\int_{0}^{1} \omega\left(Z_{(s, t)}, V_{(s, t)}\right) d s\right)
\end{aligned}
$$

We used the facts that $V_{(0, t)}=0, i\left([Z, V] \omega=L_{Z} i_{V} \omega-i_{V} L_{Z} \omega\right.$ and $L_{V} \omega=L_{V} \omega=0$.

\section{Step 3: Norm estimates}

The harmonic vector fields $\dot{\rho}_{t}$ can be written as $\dot{\rho}_{t}=\sum_{0}^{k} \lambda_{i}(t) H_{i}$, where $H_{i}=h_{i}^{\#}$ and $\left(h_{i}\right)$ is a basis of harmonic 1 -forms. Formula (19) just says:

$$
Z_{(s, t)}=\sum_{i}\left(t \lambda_{i}(s t)-2 s \int_{0}^{t} \lambda_{i}(u) d u\right) H_{i}=\sum_{i} \mu_{i}(s, t) H_{i}
$$

Hence:

$$
\begin{gathered}
\left|Z_{(s, t)}\right|=\sum_{i}\left|\mu_{i}(s, t)\right| \leq t\left|\dot{\rho}_{s t}\right|+2 s \int_{0}^{t}\left|\mathcal{H}_{t}\right| d t \\
\leq t\left|\dot{\rho}_{s t}\right|+2 s \int_{0}^{1}\left|\mathcal{H}_{t}\right| d t \leq t\left|\dot{\rho}_{s t}\right|+2 s / N .
\end{gathered}
$$

On the other hand, we have:

$$
\omega\left(Z_{(s, t)}, V_{(s, t)}\right)=\left(i_{\left(Z_{(s, t)}\right.} \omega\right)\left(V_{(s, t)}\right)=\sum_{i} \mu_{i}(s, t) h_{i}\left(V_{(s, t)}\right)
$$

Consequently:

$$
\left|\omega\left(Z_{(s, t)}, V_{(s, t)}\right)\right| \leq \sum_{i}\left|\mu_{i}(s, t) h_{i}\left(V_{(s, t)}\right)\right| .
$$

Let $\left\|h_{i}\right\|$ be the sup norm of the 1 -forms $h_{i}$, i.e $\left\|h_{i}\right\|=\sup _{x \in M} \mid\left\|h_{i}(x)\right\| \|$ and $\left\|h_{i}(x)\right\|||$ is the norm of the linear map $h_{i}(x)$ on the tangent space $T_{x} M$.

We have:

$$
\sum_{i}\left|\mu_{i}(s, t) h_{i}\left(V_{(s, t)}\right)\right| \leq\left(\sum_{i}\left|\mu_{i}(s, t)\right|\right)\left|V_{(s, t)}\right| E=\left|Z_{(s, t)}\right|\left|V_{(s, t)}\right| E
$$

where $E=\max \left\{\left\|h_{i}\right\|\right\}$.

Hence

$$
\left|w_{t}\right|=\left|\int_{0}^{1} \omega\left(Z_{(s, t)}, V_{(s, t)}\right) d s\right| \leq \int_{0}^{1}\left|\omega\left(Z_{(s, t)}, V_{(s, t)}\right)\right| d s
$$




$$
\leq E\left(\int_{0}^{1}\left(t\left|\dot{\rho}_{s t}\right|+2 s / N\right) \mid V_{s, t}\right) \mid d s
$$

Let $A=\sup _{s, t}\left|V_{s, t}\right|$, then

$$
\begin{gathered}
\left|w_{t}\right| \leq A E \int_{0}^{1}\left(t\left|\dot{\rho}_{s t}\right|+2 s / N\right) d s=A E\left(\int_{0}^{t}\left(\left|\dot{\rho}_{u}\right| d u\right)+1 / N\right) \\
\leq A E\left(\int_{0}^{1}\left(\left|\dot{\rho}_{u}\right| d u+1 / N\right) \leq 2 A E / N .\right.
\end{gathered}
$$

Therefore $\operatorname{osc}\left(w_{t}\right) \leq 4 A E / N$, hence the length of the isotopy $\rho_{t}$ is less or equal to $4 A E / N$, and therefore the Hofer norm of $\rho:\|\rho\|_{H} \leq 4 A E / N$, where $\rho=\rho_{1}$.

\section{Step 4}

Let $\mathcal{M}$ denote the space of smooth maps $c: I=[0,1] \rightarrow W$, where $W$ is the space of symplectic vector fields on $(M, \omega)$ such that $c(0)=0$ with the Hofer norm

$$
\|c\|=\int_{0}^{1}|c(t)| d t
$$

Here $|c(t)|$ is the norm given by formulas 13 and 14 .

On the space $\mathcal{M} \times I$ we define the distance $\left.d(c, s),\left(c^{\prime}, s^{\prime}\right)\right)=\left(\left(\left\|c-c^{\prime}\right\|^{2}+(s-\right.\right.$ $\left.\left.s^{\prime}\right)^{2}\right)^{1 / 2}$

Let $\mathcal{N}$ be the space of smooth functions $u: I \times I \rightarrow U$, where $U$ is the space of symplectic vector fields with the metric $\|u\|=\sup _{s, t}|u(s, t)|$.

The family of vector fields $V_{s, t}$ above is the image of $\dot{\rho}_{t}$ by the following map:

$$
\mathcal{R}: \mathcal{M} \times I \rightarrow \mathcal{N}
$$

where $\mathcal{R}=\partial_{t} \circ I_{s} \circ a_{s}$ with

$$
\begin{aligned}
& a_{s}: c(t) \mapsto t c(s t)-2 s\left(\int_{0}^{t} i(c(u) \omega d u)^{\#}\right. \\
& I_{s}: U_{s, t} \mapsto G_{s, t}: M \rightarrow M \text { where the family of diffeomorphisms } G_{s, t} \text { is obtained }
\end{aligned}
$$
by integrating in $s$ like in formula 21 .

and finally $\partial t: g_{s, t} \mapsto \partial / \partial t\left(g_{s, t}\right)$ ( formula 22$)$.

The mapping $\mathcal{R}$ is a smooth map since all its components are smooth, consequently it is Lipschitz. Therefore there is a constant $K$ such that $d\left(\mathcal{R}\left(\dot{\rho}_{t}, s\right),(0,0)\right)=$ $\sup _{s, t}\left|V_{s, t}\right| \leq K\left(\left\|\dot{\rho}_{t}\right\|^{2}+s^{2}\right)^{1 / 2}$ (Observe that $\left.\mathcal{R}(0,0)=0\right)$. 
Therefore

$$
A=\sup _{s, t}\left|V_{s, t}\right| \leq K\left((1 / N)^{2}+s^{2}\right)^{1 / 2} \leq K\left((1 / N)^{2}+1\right)^{1 / 2} \leq 2 K
$$

Finally, we get:

$$
\|\rho\|_{H} \leq\left(4 E\left(K\left((1 / N)^{2}+1\right)^{1 / 2}\right)\right) / N \leq C / N
$$

where $C=8 E K$.

Remember now that $\phi=\rho \mu$ and $\|\mu\|_{H} \leq 1 / N$. Therefore, $\|\phi\|_{H} \leq(C+11) / N$ for all $N$. Hence $\|\phi\|_{H}=0$ and consequently $\phi=i d$.

$\operatorname{Ham}(M, \omega)$ is closed in $\operatorname{Symp}(M, \omega)$

Let $\left(h_{n}\right) \in \operatorname{Ham}(M, \omega)$ be a sequence converging to $g \in \operatorname{Symp}(M, \omega)$. There exists $N_{0}$ such that for all $N \geq N_{0}$, there exists an isotopy $\Phi^{N} \in I \operatorname{so}\left(g^{-1} h_{N}\right)$ with length $l\left(\Phi^{N}\right) \leq 1 / N$. By step $1, g^{-1} h_{N}$ is hamiltonian for $N$ large. Hence $g$ is also hamiltonian.

\section{Applications to the $C^{0}$ symplectic topology}

In [10], Oh and Muller defined the group of symplectic homeomorphisms, $\operatorname{Sympeo}(M, \omega)$ as the closure of the group $\operatorname{Symp}(M, \omega)$ of $C^{\infty}$ symplectic diffeomorphisms of $(M, \omega)$ in the group Homeo $(M)$ of homeomorphisms of $M$ with the $C^{0}$ topology, and the group $\operatorname{Hameo}(M, \omega)$ of hamiltonian homeomorphisms. The group $\operatorname{Sympeo}(M \omega)$ has only $C^{0}$ topology induced from $\operatorname{Homeo}(M)$, but $\operatorname{Hameo}(M, \omega)$ has a more involved topology, called the hamiltonian topology, which combines the $C^{0}$ topology and the Hofer topology.

Using our construction, we define a symplectic topology on the space $I s o(M)$ of symplectic isotopies of $(M, \omega)$ as follows:

Fix a distance $d_{0}$ on $M$ (coming from some riemannian metric) and define the distance $\bar{d}$ on the space $\operatorname{Homeo}(M)$ of homeomorphismes of $M$ as

$$
\bar{d}(\phi, \psi)=\max \left\{d(\phi, \psi), d\left(\phi^{-1}, \psi^{-1}\right)\right\}
$$


where

$$
d(h, g)=\max _{x}\left(d_{0}(h(x), g(x))\right.
$$

for all $h, g \in$ Homeo $(M)$.

Then $(\operatorname{Homeo}(M), \bar{d})$ is a complete metric space and its metric topology is just the $C^{0}$ topology. On the space $\mathcal{P} H o m e o(M)$ of continuous paths $\lambda:[0,1] \rightarrow$ Homeo( $(M)$, we put the metric topology from the distance

$$
\bar{d}(\lambda, \mu)=\sup _{t \in[0,1]} \bar{d}(\lambda(t), \mu(t)) .
$$

We define the symplectic distance on $I s o(M)$ by:

$$
d_{\text {symp }}(\Phi, \Psi)=\bar{d}(\Phi, \Psi)+D(\Phi . \Psi)
$$

where $D$ is given by formula (5').

We call the symplectic topology on Iso( $M)$ the metric topology defined by the above distance. This topology reduces to the "hamiltonian topology" of [10] on paths in $\operatorname{Ham}(M, \omega)$.

We now define a set (which we conjecture to be a group) $\operatorname{SSympeo}(M, \omega)$ as follows: $h \in \operatorname{SSympeo}(M)$ iff there exists a continuous path $\lambda:[0,1] \rightarrow \operatorname{Homeo}(M)$ such that $\lambda(0)=i d ; \lambda(1)=h$ and a sequence $\phi_{n}^{t}$ of symplectic isotopies, which converge to $\lambda$ in the $C^{0}$ topology ( induced by the norm $\bar{d}$ ) and such that $D\left(\left(\Phi_{j}\left(\Phi_{i}\right)^{-1}\right)\right.$ tends to zero when $i$ and $j$ go to infinity. This set, which contains $\operatorname{Hameo}(M, \omega)$ will play a major role in the $C^{0}$ symplectic topology, where one may consider the subgroup of $\operatorname{Homeo}(M)$ it generates. This subgroup contains $\operatorname{Hameo}(M, \omega)$ as a normal subgroup.

\section{Final Remarks}

The metric $e$ obtained here is not an " extension" of the Hofer metric since we do not know if $e(\phi)=\|\phi\|_{H}$ when $\phi \in \operatorname{Ham}(M, \omega)$. We only know that $e(\phi) \leq\|\phi\|_{H}$. The problem of extending the Hofer norm was considered in [3]. Here we would like to make some remarks about the results of [3]. 
Extension of Oh's spectral norm. It is obvious that formulas of the extensions of the Hofer metric given in [3] give in fact extensions for any bi-invariant metric on $\operatorname{Ham}(M, \omega)$. Theorem 2 in [3] uses only the properties of bi-invariance and not the Hofer norm. Then theorem 2 of [3] can be rephrased as

\section{Theorem 2.}

Let $(M, \omega)$ be a symplectic manifold such that the homomorphism $S$ admits a continuous homomorphic right inverse, then any bi-invariant metric on $\operatorname{Ham}(M, \omega)$ extends to a right invariant metric on $\operatorname{Symp}(M, \omega)$.

Under the hypothesis of the theorem above, the spectral norm $\|.\|_{\mathcal{O}}$ of Oh extends to all of $\operatorname{Symp}(M, \omega)_{0}$. For the definition of Oh's spectral norm, we refer to [9]. An example where this hypothesis holds is $T^{2 n}$ with its natural symplectic form.

\section{Theorem 3.}

If $\Gamma=0$, Oh's spectral distance extends to $\operatorname{Symp}(M, \omega)_{0}$.

Proof.

Let $\phi_{i}, i=1,2$ two symplectomorphisms and $\Phi_{i}=\left(\phi_{t}^{i}\right) \in I s o\left(\phi_{i}\right)$. The harmonic 1-forms $\mathcal{H}\left(\Phi_{i}\right)$ depend only on $\phi_{i}$. Let $\rho_{i}$ be the time one of the 1-parameter group generated by $\mathcal{H}\left(\Phi_{i}\right)^{\#}$, then $\psi_{i}=\phi_{i} \rho_{i}^{-1} \in \operatorname{Ham}(M, \omega)$. We define the Oh distance $d_{\mathcal{O}}$ of $\phi_{1}$ and $\phi_{2}$ by:

$$
d_{\mathcal{O}}\left(\phi_{1}, \phi_{2}\right)=\left|\mathcal{H}\left(\Phi_{1}\right)-\mathcal{H}\left(\Phi_{2}\right)\right|+\left\|\psi_{1} \psi_{2}^{-1}\right\|_{\mathcal{O}}
$$

The cases where $\Gamma=0$ include oriented compact surfaces of genus bigger than one. More recently, Kedra, Kotschick and Morita [6] found a longer list of compact symplectic manifolds with vanishing flux group.

\section{References}

[1] A. Banyaga Sur la structure du groupe des difféomorphismes qui préservent une forme symplectique, Comment. Math. Helv. 53(1978) pp.174-227.

[2] A. Banyaga The structure of classical diffeomorphisms groups, Mathematics 
and its applications vol 400. Kluwer Academic Publisher's Group, Dordrecht, The Netherlands (1997).

[3] A. Banyaga , P. Donato Lengths of Contact Isotopies and Extensions of the Hofer Metric Annals of Global Analysis and Geometry 30(2006) 299-312

[4] Z. Han Bi-invariant metrics on the group of symplectomorphisms, Preprint

[5] H. Hofer On the topological properties of symplectic maps, Proc. Royal Soc. Edimburgh 115A (1990), pp.25-38

[6] J. Kedra, D. Kotschick, S. Morita Crossed flux homomorphism and vanishing theorem for flux groups Geom Funct. Anal 16(2006)no 6 1246-1273.

[7] F. Lalonde, D. McDuff The geometry of symplectic energy, Ann. Math. 141 (1995) $349-37$.

[8] F. Lalonde, L.Polterovich Symplectic diffeomorphisms as isometries of Hofer's norm Topology 36(1997) 711-727

[9] Y-G. Oh Spectral invariants, analysis of the Floer moduli space, and the geometry of hamiltonian diffeomorphisms, Duke Math. J. 130(2005) 199-295

[10] Y-G. Oh and S. Muller The group of hamiltonian homeomorphisms and $C^{0}$ symplectic topology J. Symp.Geom. to appear

[11] K. Ono Floer-Novikiv cohomology and the flux conjecture Geom. Funct. Anal.16(2006) no 5 981-1020

[12] L. Polterovich Symplectic displacement energy for Lagrangian submanifolds, Erg. Th and Dyamical Systems 13 (1993), 357-367.

[13] C. Viterbo Symplectic topology as the geometry of generating functions, Math. Annalen 292(1992), 685-710

[14] F. Warner Foundations of differentiable manifolds and Lie groups Scott, Foresman and Company (1971).

Department of Mathematics The Pennsylvania State University University Park, PA 16802 